\title{
Efficacy of the Reactive Oxygen Metabolite Test as a Predictor of Initial Heart Failure Hospitalization in Elderly Patients With Chronic Heart Failure
}

\author{
Takashi Hitsumoto
}

\begin{abstract}
Background: The reactive oxygen metabolites (d-ROMs) test has recently been explored as a novel marker of oxidative stress in vivo and used in clinical settings. Conversely, data regarding the utility of the d-ROMs test as a predictor of patients with chronic heart failure (CHF) are limited. This prospective study aims to elucidate the efficacy of the d-ROMs test as a predictor of initial heart failure (HF) hospitalization in elderly patients with CHF.
\end{abstract}

Methods: A total of 428 elderly outpatients with CHF with no history of HF hospitalization (108 males, 320 females; mean age, $75 \pm 7$ years) were enrolled. Based on the median value of d-ROMs test levels (303 U.CARR), the patients were divided into the following two groups: group L (low d-ROMs test levels) and group $\mathrm{H}$ (high d-ROMs test levels). The utility of the d-ROMs test as a predictor of initial HF hospitalization was evaluated.

Results: During the 88.1-month follow-up period, $58 \mathrm{HF}$ cases were hospitalized (group L, 17 cases; group H, 41 cases; P $<0.001$, logrank test). Multivariate Cox regression analyses revealed that group $\mathrm{H}$ exhibited a significantly higher risk for HF hospitalization than did group L (hazard ratio (HR), 2.35; 95\% confidence interval (CI), 1.37 - 4.43; P < 0.01). Furthermore, the HR (vs. group L with low brain natriuretic peptide (BNP) levels $(<200 \mathrm{pg} / \mathrm{mL}), \mathrm{HR}, 9.18 ; 95 \% \mathrm{CI}$, 4.78 - 22.94; $\mathrm{P}<0.001$ ) for the incidence of HF hospitalization increased in group $\mathrm{H}$ with high BNP levels $(\geq 200 \mathrm{pg} / \mathrm{mL})$.

Conclusions: The present study demonstrates that high d-ROMs test levels predict initial HF hospitalization in elderly patients with CHF. In addition, the predictive value for the incidence of HF hospitalization increases by using a combination of two biomarkers as d-ROMs test and BNP levels.

Keywords: Reactive oxygen metabolites; Chronic heart failure; Heart failure hospitalization; Brain natriuretic peptide; Cardio-ankle vascular index; $\beta$-blocker

Manuscript submitted May 18, 2018, accepted May 25, 2018

Hitsumoto Medical Clinic, 2-7-7, Takezakicyou, Shimonoseki City, Yamaguchi, 750-0025, Japan. Email: thitsu@jcom.home.ne.jp

doi: https://doi.org/10.14740/cr733w

\section{Introduction}

Recently, a global upsurge has been observed in the incidence of chronic heart failure (CHF) because of extended life expectancy $[1,2]$. In reality, several elderly patients with CHF, including asymptomatic phases, exist in daily practice. Furthermore, epidemiological studies have suggested that patients with CHF with a history of HF hospitalization exhibit poor prognosis because of re-hospitalization owing to HF, cardiovascular diseases, or other adverse events $[3,4]$. Thus, it is important to consider the adequate diagnosis and therapy for elderly patients with CHF before the incidence of HF hospitalization.

Several fundamental and clinical studies have reported that oxidative stress plays a vital role in the pathophysiology of CHF [5-8]. Among the oxidative stress markers in vivo, the reactive oxygen metabolite (d-ROMs) test, which reflects the amount of hydroperoxide, is simply assessed using serum samples in clinical settings. In addition, clinical studies have suggested a correlation between the d-ROMs test and cardiovascular risk factors or cardiovascular disease [9-11]. By contrast, data regarding the correlation between the d-ROMs test and the pathogenesis or significance of predictors for patients with CHF are limited $[12,13]$. To the best of our knowledge, to date, no study has assessed the significance of the d-ROMs test as a predictor for initial HF hospitalization in patients with CHF. Thus, this prospective study aimed to elucidate the utility of the d-ROMs test as a predictor for initial HF hospitalization in elderly patients with CHF.

\section{Materials and Methods}

\section{Patients}

Totally, 428 elderly outpatients with CHF (age, $\geq 65$ years) with no history of HF hospitalization were enrolled between November 2007 and November 2009 at the Hitsumoto Medical Clinic, Yamaguchi, Japan. CHF was defined according to the ACC/AHA 2005 Guidelines for the Diagnosis and Management of Heart Failure in Adults [14]. The enrolled patients included $108(25.2 \%)$ males and $320(74.8 \%)$ females; and mean age, $75 \pm 7$ years (mean \pm standard deviation). Based 
on the median value of d-ROMs test levels (303 U.CARR), the patients were assigned to one of the following two groups: group L (low d-ROMs test levels) or group H (high d-ROMs test levels). The study protocol was approved by our Local Ethics Committee, and informed consent was obtained from all participants.

\section{D-ROMs test measurement}

Blood samples were collected from the brachial vein of all patients; the blood was collected in the morning, after an overnight fast, but before breakfast. The d-ROMs test was conducted by the Free Radical Analytical System (Diacron, Grosseto, Italy), as reported previously [15]. Briefly, in a pipette, $25 \mu \mathrm{L}$ of the serum sample was mixed with an acetic acid buffered solution ( $\mathrm{pH} 4.8$ ) to fix the hydrogen ion concentration; a chromogenic substrate was then added to the mixture. In an acidified medium, both bivalent and trivalent iron from the blood protein ionize and work as catalysts to break down the hydroperoxide component in the serum into alkoxyl and peroxy radicals to become free radicals. Next, the mixture was incubated in the thermostatic block of the system followed by its transfer into a cuvette, including colorless chromogen. Subsequently, the chromogen was oxidized by free radicals to a radical cation, which was magenta in color. The intensity of the magenta color reflects the concentration of hydroperoxide in the serum; this was measured using a photometer after centrifuging the prepared sample for $1 \mathrm{~min}$. The measured value was expressed as U.CARR ( $\left.1 \mathrm{U} . C A R R=0.08 \mathrm{mg} / \mathrm{dL} \mathrm{H}_{2} \mathrm{O}_{2}\right)$. A previous study suggested that the accuracy of this method was established (intra- and inter-assay coefficients of variation, $<$ $3 \%)[16]$.

\section{Evaluation of clinical parameters}

The present study evaluated various clinical parameters, such as classic risk factors for cardiovascular diseases, history of ischemic heart disease, echocardiographic findings, hemoglobin concentration, kidney function, brain natriuretic peptide (BNP) levels, high-sensitivity C-reactive protein (hs-CRP) levels, and arterial stiffness. Obesity was identified using the body mass index, calculated as weight $(\mathrm{kg})$ divided by the squared height $\left(\mathrm{m}^{2}\right)$. Current smoking was defined as smoking at least one cigarette per day over the previous 28 days. A history of ischemic heart disease was defined as patients with a history of myocardial infarction and/or angiography-proven significant stenosis. Hypertension was defined as systolic blood pressure $\geq 140 \mathrm{~mm} \mathrm{Hg}$, diastolic blood pressure $\geq 90 \mathrm{~mm} \mathrm{Hg}$, or administration of antihypertensive medications. Dyslipidemia was defined as low-density lipoprotein cholesterol levels $\geq$ $140 \mathrm{mg} / \mathrm{dL}$, high-density lipoprotein cholesterol levels $\leq 40$ $\mathrm{mg} / \mathrm{dL}$, triglyceride levels $\geq 150 \mathrm{mg} / \mathrm{dL}$, or ongoing treatment for dyslipidemia. Diabetes mellitus was defined as a fasting blood glucose level greater than or equal to $126 \mathrm{mg} / \mathrm{dL}$ or the use of antidiabetic treatment. The standard technique for echocardiography was performed using a commercial device (UF850XTD; Fukuda Denshi, Tokyo, Japan). Valvular heart dis- ease comprised aortic or mitral valve disease (aortic stenosis, aortic regurgitation, mitral stenosis, and mitral regurgitation). Left ventricular wall thickness, extended period diameter, ejection fraction, and left atrial dimension were also assessed using echocardiography. The estimated glomerular filtration rate (eGFR) was evaluated using the "adjusted modification of diet in the renal disease study equation", as proposed by the working group of the Japanese Chronic Kidney Disease Initiative [17]. BNP levels were measured using a commercial kit (SHIONOSPOT Reader; Shionogi \& Co., Osaka, Japan) and the hs-CRP concentration using high-sensitivity latexenhanced immunonephelometric assay. Cardio-ankle vascular index (CAVI) was measured as a physiological marker of arterial stiffness using a VaSera CAVI instrument (Fukuda Denshi), as described previously [18]. Briefly, brachial and ankle pulse waves were determined using inflatable cuffs, with the pressure maintained between 30 and $50 \mathrm{~mm} \mathrm{Hg}$ to ensure that the cuff pressure exerted minimal effects on systemic hemodynamics. Of note, systemic blood and pulse pressures were simultaneously determined, with the participants in the supine position. Besides, CAVI was measured after a 10 -min rest in a quiet room. The accuracy of CAVI is suggested to be less in the presence of non-sinus rhythm or obstructive arteriosclerosis; thus, we excluded patients with chronic atrial fibrillation and/or obstructive arteriosclerosis (ankle-brachial index $<$ $0.9)$. The mean values of the left and right sides were used for statistically evaluating the CAVI.

\section{Patient follow-up}

The follow-up period was terminated in November 2017. The endpoint for this study was HF hospitalization. All patients were followed-up for a median of 81.1 months (range, 6 - 120 months) to determine the incidence of HF hospitalization.

\section{Statistical analysis}

Data were analyzed using the Stat View-J 5.0 (HULINKS, Tokyo, Japan) and MedCalc for Windows (version 14.8.1; MedCalc Software, Ostend, Belgium). Data are presented as mean \pm standard deviation. Between-group comparisons were performed using the Student's $t$-test or the Mann-Whitney U-test. In addition, the event-free survival rate curves were calculated using the Kaplan-Meier analysis and the differences between the curves were evaluated using the long-rank test. Furthermore, multivariate analysis was performed using the multivariate Cox regression analysis. Receiver operating characteristics (ROC) curves were constructed and Youden Index was used to determine optimal cut-offs for predicting the HF hospitalization. $\mathrm{P}<0.05$ was considered statistically significant.

\section{Results}

Table 1 presents the characteristics of all patients at registration. Mean d-ROMs levels of groups L and $\mathrm{H}$ were 235 and 
Table 1. Characteristics of Patients

\begin{tabular}{|c|c|c|c|c|}
\hline & Overall & Group L & Group H & P value \\
\hline N (Male/female) & $428(108 / 320)$ & $214(58 / 156)$ & $214(50 / 164)$ & 0.375 \\
\hline Age (yrs) & $75 \pm 7$ & $74 \pm 6$ & $76 \pm 8$ & 0.108 \\
\hline Body mass index & $22.8 \pm 3.5$ & $22.9 \pm 3.4$ & $22.8 \pm 3.6$ & 0.546 \\
\hline Current smoker, n (\%) & $99(23)$ & $46(21)$ & $53(25)$ & 0.423 \\
\hline Systolic blood pressure (mm Hg) & $144 \pm 24$ & $144 \pm 21$ & $144 \pm 27$ & 0.852 \\
\hline Diastolic blood pressure (mm Hg) & $86 \pm 11$ & $86 \pm 11$ & $86 \pm 10$ & 0.695 \\
\hline Pulse rate (/min) & $65 \pm 13$ & $64 \pm 12$ & $66 \pm 14$ & 0.363 \\
\hline Dyslipidemia, n (\%) & $224(52)$ & $102(48)$ & $122(57)$ & 0.051 \\
\hline LVDd (mm) & $48 \pm 4$ & $48 \pm 3$ & $48 \pm 4$ & 0.529 \\
\hline LVEF (\%) & $67.8 \pm 11.7$ & $67.4 \pm 12.1$ & $68.3 \pm 11.3$ & 0.3 \\
\hline $\mathrm{LAD}(\mathrm{mm})$ & $42 \pm 6$ & $42 \pm 6$ & $43 \pm 6$ & 0.421 \\
\hline Hemoglobin (g/dL) & $12.7 \pm 1.6$ & $12.8 \pm 1.6$ & $12.7 \pm 1.5$ & 0.592 \\
\hline $\mathrm{eGFR}\left(\mathrm{mL} / \mathrm{min} / 1.73 \mathrm{~m}^{2}\right)$ & $55.2 \pm 22.2$ & $55.6 \pm 22.4$ & $54.8 \pm 21.9$ & 0.742 \\
\hline Log-BNP (pg/mL) & $2.0 \pm 0.4$ & $1.9 \pm 0.4$ & $2.1 \pm 0.3$ & $<0.01$ \\
\hline hs-CRP (mg/dL) & $-1.2 \pm 0.5$ & $-1.3 \pm 0.5$ & $-1.1 \pm 0.5$ & $<0.05$ \\
\hline CAVI & $9.5 \pm 1.2$ & $9.3 \pm 1.2$ & $9.8 \pm 1.2$ & $<0.001$ \\
\hline \multicolumn{5}{|l|}{ Medication } \\
\hline
\end{tabular}

Continuous values are mean \pm SD. D-ROMs: derivatives of reactive oxygen metabolites; IVSTd: interventricular septal thickness at end-diastole; LVDd: left ventricular end-diastolic diameter; LVEF: left ventricular ejection fraction; LAD: left atrial dimension; eGFR: estimated glomerular filtration rate; BNP: brain natriuretic peptide; hs-CRP: high sensitivity $\mathrm{C}$ reactive protein; CAVI: cardio-ankle vascular index; RAS: renin-angiotensin system.

421 U.CARR, respectively. The presence of diabetes mellitus, BNP levels, hs-CRP levels, and CAVI was considerably higher in group $\mathrm{H}$ than in group L. Figure 1 shows the Kaplan-Meier curve for the incidence of HF hospitalization. During the 88.1-month follow-up period, $58 \mathrm{HF}$ hospitalization cases were reported (group L, 17 cases; group H, 41 cases). The Kaplan-Meier curve demonstrated that group $\mathrm{H}$ exhibited a significantly higher HF hospitalization incidence than did group L (log-rank test, $\mathrm{P}<0.001)$. Table 2 presents the clinical parameters at registration of all patients with and without HF hospitalization. In addition, age, smoking status, presence of diabetes mellitus, BNP levels, hs-CRP levels, and CAVI were considerably higher, and the eGFR, renin-angiotensin system inhibitor use, and $\beta$-blocker use were considerably lower in patients with HF hospitalization than in those without HF hospitalization. Table 3 presents the results of the multivariate Cox regression analysis for the HF hospitalization incidence.

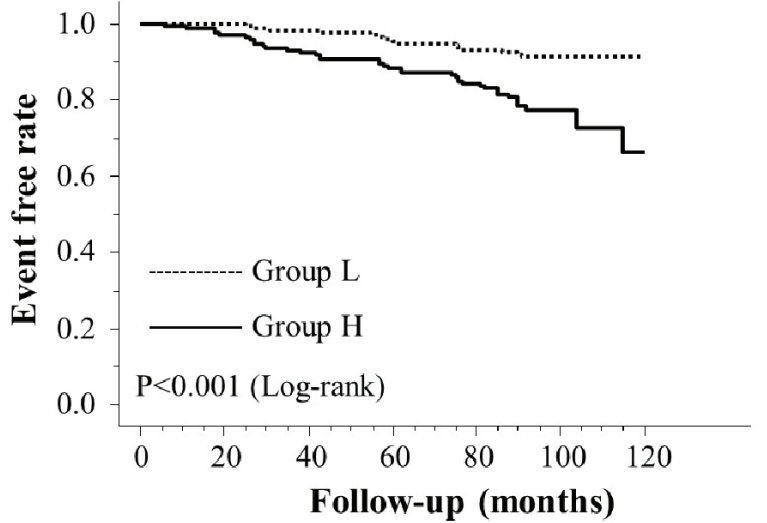

Figure 1. Kaplan-Meier curve for the incidence of heart failure hospitalization. 
Table 2. Clinical Parameters at Registration of Patients With and Without Heart Failure Hospitalization

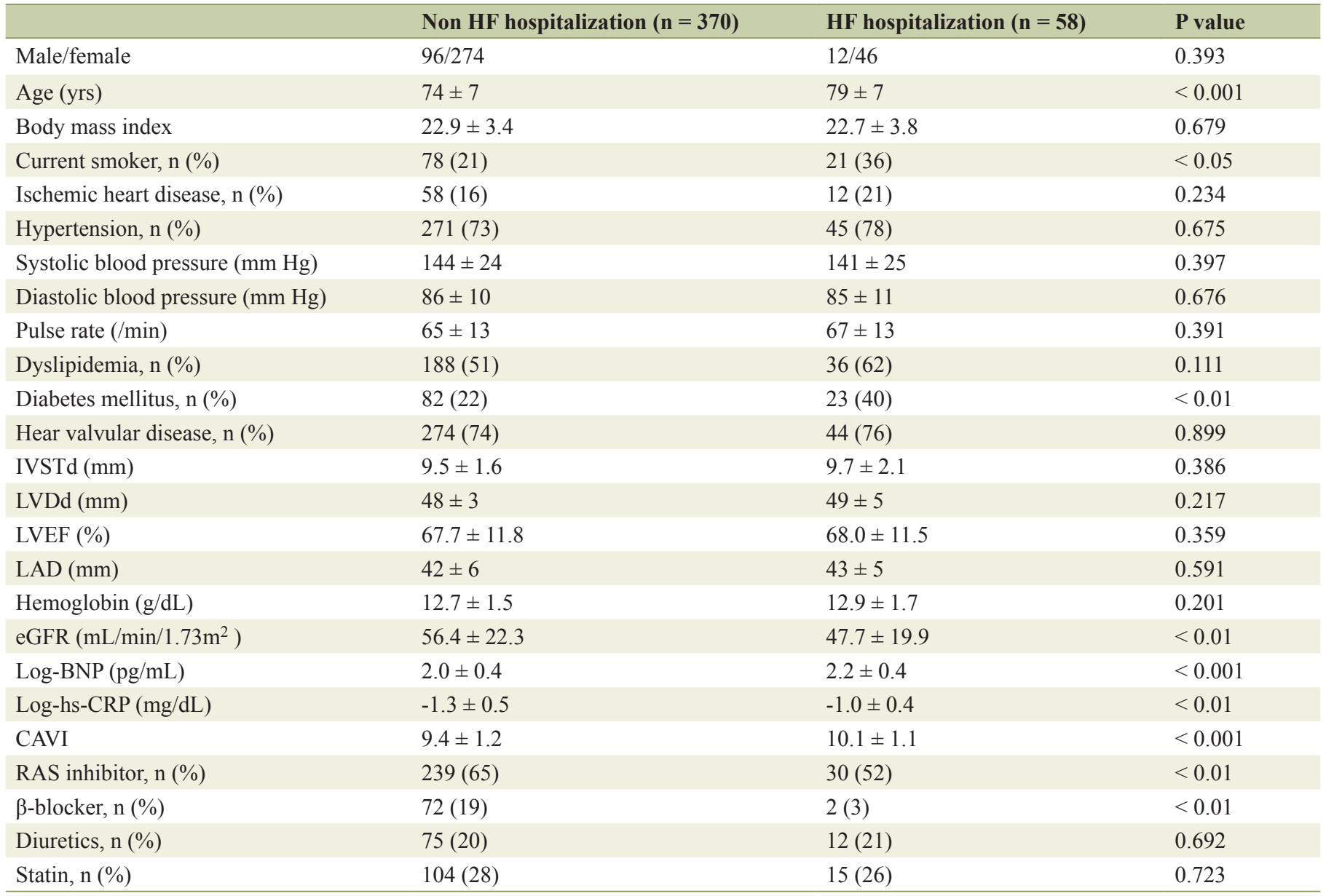

Continuous values are mean \pm SD. HF: heart failure; IVSTd: interventricular septal thickness at end- diastole; LVDd: left ventricular end-diastolic diameter; LVEF: left ventricular ejection fraction; LAD: left atrial dimension; eGFR: estimated glomerular filtration rate; BNP: brain natriuretic peptide; hs-CRP: high sensitivity $\mathrm{C}$ reactive protein; CAVI: cardio-ankle vascular index; RAS: renin-angiotensin system.

Table 3. Multivariate Cox Regression Analysis for Heart Failure Hospitalization

\begin{tabular}{llll}
\hline & HR & $\mathbf{9 5 \%}$ CI & P value \\
\hline Age $(\geq 75$ years $)$ & 2.78 & $1.53-5.10$ & $<0.01$ \\
Group H & 2.35 & $1.37-4.43$ & $<0.01$ \\
BNP $(\geq 200 \mathrm{pg} / \mathrm{mL})$ & 2.22 & $1.21-3.52$ & $<0.01$ \\
Diabetes mellitus & 1.94 & $1.06-3.38$ & $<0.05$ \\
CAVI $(\geq 10)$ & 1.82 & $1.07-3.19$ & $<0.05$ \\
$\beta$-blocker & 0.52 & $0.33-0.98$ & $<0.05$ \\
RAS inhibitor & 0.44 & $0.27-1.11$ & 0.089 \\
eGFR $\left(<60 \mathrm{~mL} / \mathrm{min} / 1.73 \mathrm{~m}^{2}\right)$ & 1.53 & $0.85-2.77$ & 0.159 \\
hs-CRP $(\geq 0.1 \mathrm{mg} / \mathrm{dL})$ & 1.41 & $0.79-2.53$ & 0.190 \\
Current smoker & 1.31 & $0.84-1.73$ & 0.204 \\
\hline
\end{tabular}

HR: hazard ratio; Cl: confidence interval; BNP: brain natriuretic peptide; CAVI: cardio-ankle vascular index; RAS: renin- angiotensin system; eGFR: estimated glomerular filtration rate; hs-CRP: high sensitivity $\mathrm{C}$ reactive protein.
Ten variables were selected based on significant factors for $\mathrm{HF}$ hospitalization in the univariate analysis. Of these, six variables (age, group H, BNP levels, diabetes mellitus, CAVI, and $\beta$-blocker use) exhibited a considerable hazard ratio (HR) for the HF hospitalization incidence. The patients were divided into four groups based on their d-ROMs test and BNP levels to clarify the clinical efficacy of a combination of two biomarkers as the d-ROMs test and BNP levels, and a multivariate Cox regression analysis was performed (Fig. 2). Besides, group $\mathrm{H}$ with low BNP levels $(<200 \mathrm{pg} / \mathrm{mL})$ or group L with high BNP levels $(\geq 200 \mathrm{pg} / \mathrm{mL}$ ) exhibited significantly higher HR (HR: 2.21, 95\% CI: 1.06 - 4.71, P < 0.05; HR: 2.29, 95\% CI: 1.09 $5.93, \mathrm{P}<0.05$, respectively) than group $\mathrm{L}$ with low BNP levels. Furthermore, HR of group H with high BNP levels compared with group L with low BNP levels exhibited higher HR (HR, 9.18; 95\% CI, $4.78-22.94 ; \mathrm{P}<0.001)$ than the other groups. Figure 3 shows the ROC curve analysis for the incidence of HF hospitalization using d-ROMs test. The d-ROMs test levels cut-off 319 U. CARR yielded the largest area under the curve of 0.701 (95\%CI: $0.655-0.744)$, with a sensitivity of $86.4 \%$ and specificity of $51.8 \%$ for discriminating between non-hos- 


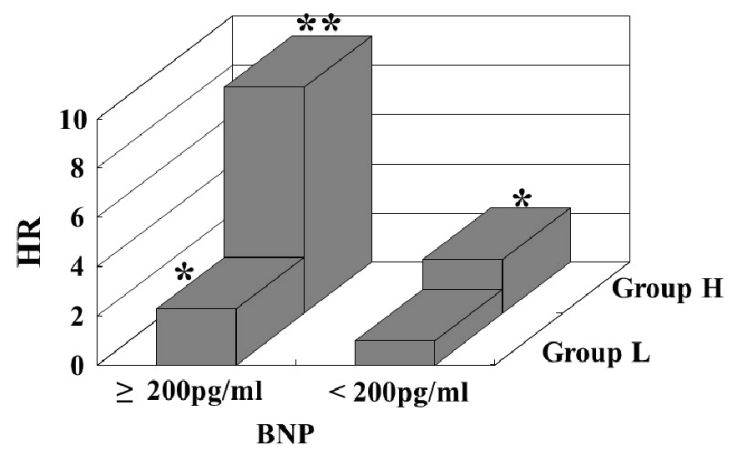

Figure 2. Multivariate Cox regression analysis for heart failure hospitalization using a combination of the d-ROMs test and BNP. Group $\mathrm{H}$ with low BNP levels $(<200 \mathrm{pg} / \mathrm{mL})$ or group $L$ with high BNP levels $(\geq 200 \mathrm{pg} / \mathrm{mL}$ ) exhibited significantly higher HR (HR: $2.21,95 \% \mathrm{Cl}: 1.06$ - 4.71, P < 0.05; HR: 2.29, 95\% Cl: 1.09 - 5.93, P < 0.05, respectively) than group $L$ with low BNP levels. Furthermore, HR of group $H$ with high BNP levels compared with group $L$ with low BNP levels exhibited higher $\mathrm{HR}(\mathrm{HR}, 9.18 ; 95 \% \mathrm{Cl}, 4.78-22.94 ; \mathrm{P}<0.001)$ than the other groups. Adjustment factors are age, diabetes mellitus, CAVI, and $\beta$-blocker use. ${ }^{*} P<0.05$ vs. group $L$ with low BNP levels; ${ }^{*} P<0.001$ vs. group $L$ with low BNP levels. HR: hazard ratio, $\mathrm{Cl}$ : confidence interval, d-ROMs: derivatives of reactive oxygen metabolites; BNP: brain natriuretic peptide; HR: hazard ratio; CAVI: cardio-ankle vascular index.

pitalization and hospitalization at follow up period.

\section{Discussion}

Previous studies have reported a correlation between aging, diabetes mellitus, and HF incidence $[1,2,19]$. Likewise, this study also suggested that these factors were selected as a predictor for initial HF hospitalization in elderly patients with CHF. In addition, this study suggested that the d-ROMs test, as a novel marker for oxidative stress in vivo, was a predictor for initial HF hospitalization. Moreover, BNP, CAVI as a novel marker for arterial stiffness, and $\beta$-blocker use were selected as a predictor for initial HF hospitalization, and the clinical efficacy of using a combination of the d-ROMs test and BNP as a predictor for initial HF hospitalization was indicated in this study.

A recent study highlighted the importance of $\mathrm{CHF}$, which preserved the left ventricular ejection fraction (HFPEF) [20]. Left ventricular diastolic dysfunction is considered the leading cause of HFPEF, and animal or human studies have suggested that oxidative stress causes left ventricular diastolic dysfunction $[21,22]$. By contrast, the mean value of the left ventricular ejection fraction levels in this study was $67.8 \%$, suggesting that this feature of $\mathrm{CHF}$ is considered the reflecting pathogenesis of HFPEF. Hirata et al reported the clinical efficacy of d-ROMs levels as a predictor for cardiovascular events in patients with HFPEF with a history of HF hospitalization. In addition, they reported that compared with patients with low levels, patients with high d-ROMs levels (> 346 U.CARR), which are divided by the median value, exhibited considerably higher cardiovascular events, including HF re-hospitalization [13]. In the contrary, the present study suggested that patients

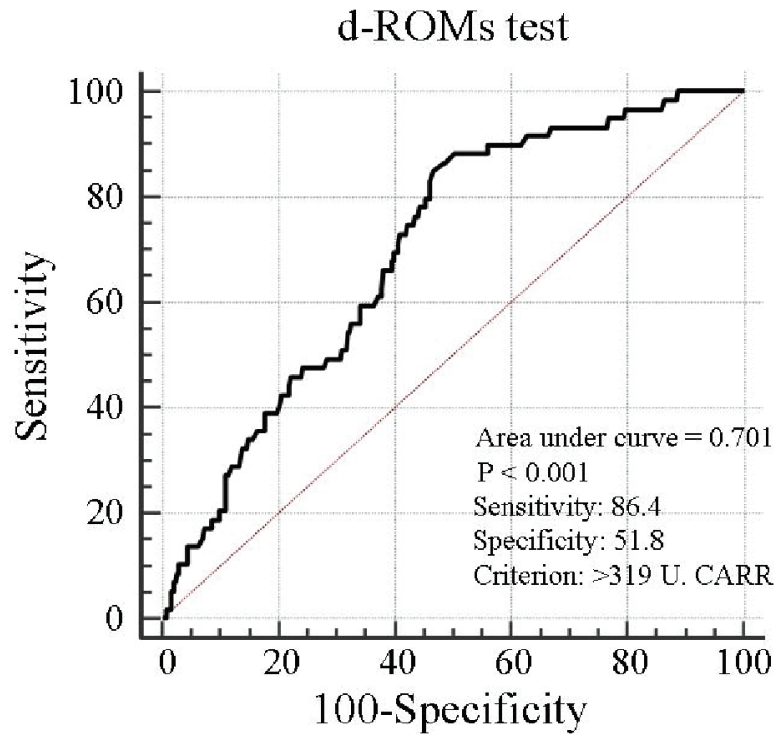

Figure 3. Prediction of heart failure hospitalization at follow-up period using d-ROMs test. The d-ROMs test levels cut-off 319 U. CARR yielded the largest area under the curve of $0.701(95 \% \mathrm{Cl}: 0.655-0.744)$, with a sensitivity of $86.4 \%$ and specificity of $51.8 \%$ for discriminating between non-hospitalization and hospitalization at follow-up period. $\mathrm{Cl}$ : confidence interval; d-ROMs: derivatives of reactive oxygen metabolites.

with high d-ROMs levels (> 303 U.CARR), which are also divided by the median value, exhibited a considerably higher initial HF hospitalization incidence than patients with low dROMs levels. In addition, ROC curve analysis indicated that d-ROMs test as 319 U. CARR was an optimal cut-off value to predict the incidence of HF hospitalization. Thus, d-ROMs test as $319 \mathrm{U}$. CARR is considered as appropriate target value in order to diagnose or predict initial HF hospitalization in elderly CHF patients with no history of HF hospitalization.

Recent clinical studies have reported that the $\beta$-blocker is clinically useful for not only CHF with a reduced left ventricular ejection fraction but also CHF with HFPEF [23, 24]. In addition, the results indicated that the $\beta$-blocker was selected as an inhibitory factor for initial HF hospitalization. Conversely, the incidence of the use of the $\beta$-blocker in patients with high d-ROMs levels was as low as $15 \%$. Moreover, patients with initial HF hospitalization incidence exhibited only $3 \%$ use of the $\beta$-blocker. Some basic and clinical studies reported that $\beta$-blocker inhibits oxidative stress in myocardial cells or improves left ventricular diastolic function $[25,26]$. Thus, additional therapy using the $\beta$-blocker for patients with high $\mathrm{d}$ ROMs levels with no administration of $\beta$-blocker is required, consequently expecting to prevent the incidence of initial HF hospitalization.

The clinical utility of BNP in CHF diagnosis or therapy is well-established [27]. This study suggested that high BNP levels were selected as a predictor for initial HF hospitalization. Conversely, several cut-off BNP levels were reported for cardiovascular disease incidence, including HF hospitalization [28-30]. In addition, Fischer et al reported that the BNP levels were different when measured using the kit [31]. However, 
this study suggested that the predictive value of BNP levels, which is measured using the SHIONOSPOT Reader as a commercial kit for the incidence of initial HF hospitalization, was $200 \mathrm{pg} / \mathrm{mL}$ degrees in elderly patients with CHF. In this study, all participants were divided into four groups based on their d-ROMs test levels (303 U.CARR) and BNP levels (200 pg/ $\mathrm{mL}$ ) to clarify the clinical efficacy of using a combination of two biomarkers as d-ROMs test and BNP levels. Thus, a multivariate analysis was performed. The HR of patients with both high d-ROMs and high BNP levels compared with patients with both low d-ROMs and low BNP levels exhibited a HR with approximately four points higher than the HR of patients with high d-ROMs or high BNP levels alone. Because both the d-ROMs test and BNP levels can be measured in a short duration of approximately $15 \mathrm{~min}$ in clinical settings, a speedy and effective diagnosis and treatment for patients with $\mathrm{CHF}$ was expected using a combination of the d-ROMs test and BNP in daily practice.

CAVI is a marker of systemic arterial stiffness and is independently associated with blood pressure levels [18]. Conversely, there exist several studies concerning the relationship between the physiological marker of arterial stiffness and the pathogenesis or prognosis of HF [32-34]. Meguro et al reported that high brachial-ankle pulse wave velocity level, which is a marker of arterial stiffness, predicts HF re-hospitalization in patients with $\mathrm{CHF}$ [33]. In addition, this study suggested that arterial stiffness is an essential factor for initial HF hospitalization and that a CAVI value $\geq 10$ is considered the predictive value for the incidence of initial HF hospitalization in elderly patients with CHF. By contrast, this study also demonstrated that patients with high d-ROMs levels exhibited a considerably higher CAVI than those with low d-ROMs levels did. Several clinical studies have reported that medications, such as antihypertensive, antihyperlipidemic, and antidiabetic drugs, decrease CAVI [35-37]. Moreover, some studies have reported the clinical utility of these drugs in improving oxidative stress in vivo [38-40]. Miyashita et al reported that a decrease in urinary 8-hydroxydeoxy-guanosine, one of the markers of oxidative stress in vivo, exhibited a marked positive correlation with a decrease in CAVI by intervention therapy using statin or angiotensin receptor blocker $[35,36]$. However, Iwasa et al reported that an improvement in the physical activity exhibited an independent correlation with an improvement in CAVI [41]. Thus, a decrease in the incidence of initial HF hospitalization in elderly patients with CHF has a possibility by interventions, such as medications with antioxidant effects or the improvement in the physical activity for target CAVI levels $<10$.

\section{Limitations}

This study has several limitations. First, this study was conducted at a single-center with a relatively small sample size. Thus, the findings cannot be generalized to all medical centers. Second, only one point of the d-ROMs test levels was measured at registration. Thus, additional examination regarding the correlation between a serial change in the d-ROMs test and HF hospitalization is required. Finally, further studies concerning patients with high d-ROMs test levels are warranted to determine whether aggressive antioxidative stress therapy reduces the incidence of initial HF hospitalization in elderly patients with CHF.

\section{Conclusions}

This study demonstrates that high d-ROMs test levels predict initial HF hospitalization in elderly patients with CHF. In addition, the predictive value for the incidence of HF hospitalization increases by using a combination of two biomarkers as d-ROMs test and BNP levels. Nevertheless, further studies concerning patients with high d-ROMs test levels are warranted to determine whether aggressive antioxidative stress therapy reduces the incidence of initial HF hospitalization in elderly patients with $\mathrm{CHF}$.

\section{Conflict of Interest}

The author has reported no conflict of interest.

\section{Grant Support}

None.

\section{References}

1. Ho KK, Pinsky JL, Kannel WB, Levy D. The epidemiology of heart failure: the Framingham Study. J Am Coll Cardiol. 1993;22(4 Suppl A):6A-13A.

2. Eriksson H, Svardsudd K, Larsson B, Ohlson LO, Tibblin G, Welin L, Wilhelmsen L. Risk factors for heart failure in the general population: the study of men born in 1913. Eur Heart J. 1989;10(7):647-656.

3. Tsuchihashi M, Tsutsui H, Kodama K, Kasagi F, Takeshita A. Clinical characteristics and prognosis of hospitalized patients with congestive heart failure - a study in Fukuoka, Japan. Jpn Circ J. 2000;64(12):953-959.

4. Ruigomez A, Michel A, Martin-Perez M, Garcia Rodriguez LA. Heart failure hospitalization: An important prognostic factor for heart failure re-admission and mortality. Int J Cardiol. 2016;220:855-861.

5. Li SY, Yang X, Ceylan-Isik AF, Du M, Sreejayan N, Ren J. Cardiac contractile dysfunction in Lep/Lep obesity is accompanied by NADPH oxidase activation, oxidative modification of sarco(endo)plasmic reticulum Ca2+-ATPase and myosin heavy chain isozyme switch. Diabetologia. 2006;49(6):1434-1446.

6. Ide T, Tsutsui H, Hayashidani S, Kang D, Suematsu N, Nakamura K, Utsumi H, et al. Mitochondrial DNA damage and dysfunction associated with oxidative stress in failing hearts after myocardial infarction. Circ Res. 2001;88(5):529-535.

7. Kobayashi S, Susa T, Tanaka T, Wada Y, Okuda S, Doi M, Nao T, et al. Urinary 8-hydroxy-2'-deoxyguanosine 
reflects symptomatic status and severity of systolic dysfunction in patients with chronic heart failure. Eur J Heart Fail. 2011;13(1):29-36.

8. Savic-Radojevic A, Pljesa-Ercegovac M, Matic M, Simic D, Radovanovic S, Simic T. Novel Biomarkers of Heart Failure. Adv Clin Chem. 2017;79:93-152.

9. Kotani K, Tsuzaki K, Taniguchi N, Sakane N. Correlation between reactive oxygen metabolites \& atherosclerotic risk factors in patients with type 2 diabetes mellitus. Indian J Med Res. 2013;137(4):742-748.

10. Masaki N, Sato A, Horii S, Kimura T, Toya T, Yasuda R, Namba T, et al. Usefulness of the d-ROMs test for prediction of cardiovascular events. Int $\mathrm{J}$ Cardiol. 2016;222:226-232.

11. Hitsumoto T. Relationship between hemorheology assessed using microchannel array flow analyzer and kidney function in hypertensive patients. Cardiol Res. 2017;8(4):147-153.

12. Hitsumoto T. Clinical significance of skin autofluorescence in patients with type 2 diabetes mellitus with chronic heart failure. Cardiol Res. 2018;9(2):83-89.

13. Hirata Y, Yamamoto E, Tokitsu T, Kusaka H, Fujisue K, Kurokawa H, Sugamura K, et al. Reactive oxidative metabolites are associated with the severity of heart failure and predict future cardiovascular events in heart failure with preserved left ventricular ejection fraction. Int J Cardiol. 2015;179:305-308.

14. Hunt SA, American College of C, American Heart Association Task Force on Practice G. ACC/AHA 2005 guideline update for the diagnosis and management of chronic heart failure in the adult: a report of the American College of Cardiology/American Heart Association Task Force on Practice Guidelines (Writing Committee to Update the 2001 Guidelines for the Evaluation and Management of Heart Failure). J Am Coll Cardiol. 2005;46(6):e1-82.

15. Cesarone MR, Belcaro G, Carratelli M, Cornelli U, De Sanctis MT, Incandela L, Barsotti A, et al. A simple test to monitor oxidative stress. Int Angiol. 1999;18(2):127-130.

16. Verde V, Fogliano V, Ritieni A, Maiani G, Morisco F, Caporaso N. Use of N,N-dimethyl-p-phenylenediamine to evaluate the oxidative status of human plasma. Free Radic Res. 2002;36(8):869-873.

17. Imai E, Horio M, Nitta K, Yamagata K, Iseki K, Hara $\mathrm{S}$, Ura N, et al. Estimation of glomerular filtration rate by the MDRD study equation modified for Japanese patients with chronic kidney disease. Clin Exp Nephrol. 2007;11(1):41-50.

18. Shirai K, Utino J, Otsuka K, Takata M. A novel blood pressure-independent arterial wall stiffness parameter; cardio-ankle vascular index (CAVI). J Atheroscler Thromb. 2006;13(2):101-107.

19. Kannel WB, McGee DL. Diabetes and cardiovascular disease. The Framingham study. JAMA. 1979;241(19):20352038.

20. Zile MR, Baicu CF, Gaasch WH. Diastolic heart failureabnormalities in active relaxation and passive stiffness of the left ventricle. N Engl J Med. 2004;350(19):19531959.

21. Koncsos G, Varga ZV, Baranyai T, Boengler K, Rohrbach
S, Li L, Schluter KD, et al. Diastolic dysfunction in prediabetic male rats: Role of mitochondrial oxidative stress. Am J Physiol Heart Circ Physiol. 2016;311(4):H927H943.

22. Masugata $H$, Senda $S$, Inukai $M$, Himoto $T$, Imachi $H$, Murao K, Hosomi N, et al. Association between oxidative stress assessed by urinary 8-hydroxydeoxyguanosine and the cardiac function in hypertensive patients without overt heart disease. Clin Exp Hypertens. 2013;35(5):308312.

23. Garcia-Egido A, Andrey JL, Puerto JL, Aranda RM, Pedrosa MJ, Lopez-Saez JB, Rosety M, et al. Beta-blocker therapy and prognosis of heart failure patients with newonset diabetes mellitus. Int J Clin Pract. 2015;69(5):550559.

24. Ruiz G, Andrey JL, Puerto JL, Escobar MA, Romero SP, Aranda R, Pedrosa MJ, et al. Prognosis of heart failure with preserved ejection fraction treated with betablockers: A propensity matched study in the community. Int J Cardiol. 2016;222:594-602.

25. Nakamura K, Murakami M, Miura D, Yunoki K, Enko K, Tanaka M, Saito Y, et al. Beta-Blockers and Oxidative Stress in Patients with Heart Failure. Pharmaceuticals (Basel). 2011;4(8):1088-1100.

26. Dogan A, Karabacak M, Tayyar S, Erdogan D, Ozaydin M. Comparison of the effects of carvedilol and nebivolol on diastolic functions of the left ventricle in patients with non-ischemic heart failure. Cardiol J. 2014;21(1):76-82.

27. Berger R, Moertl D, Peter S, Ahmadi R, Huelsmann M, Yamuti S, Wagner B, et al. N-terminal pro-B-type natriuretic peptide-guided, intensive patient management in addition to multidisciplinary care in chronic heart failure a 3-arm, prospective, randomized pilot study. J Am Coll Cardiol. 2010;55(7):645-653.

28. Maeda K, Tsutamoto T, Wada A, Mabuchi N, Hayashi M, Tsutsui T, Ohnishi M, et al. High levels of plasma brain natriuretic peptide and interleukin- 6 after optimized treatment for heart failure are independent risk factors for morbidity and mortality in patients with congestive heart failure. J Am Coll Cardiol. 2000;36(5):1587-1593.

29. Nishii $M$, Inomata $T$, Takehana $H$, Naruke $T$, Yanagisawa T, Moriguchi M, Takeda S, et al. Prognostic utility of B-type natriuretic peptide assessment in stable low-risk outpatients with nonischemic cardiomyopathy after decompensated heart failure. J Am Coll Cardiol. 2008;51(24):2329-2335.

30. Porapakkham P, Porapakkham P, Zimmet H, Billah B, Krum H. B-type natriuretic peptide-guided heart failure therapy: A meta-analysis. Arch Intern Med. 2010;170(6):507-514.

31. Fischer Y, Filzmaier K, Stiegler H, Graf J, Fuhs S, Franke A, Janssens U, et al. Evaluation of a new, rapid bedside test for quantitative determination of B-type natriuretic peptide. Clin Chem. 2001;47(3):591-594.

32. Yambe M, Tomiyama H, Hirayama Y, Gulniza Z, Takata Y, Koji Y, Motobe K, et al. Arterial stiffening as a possible risk factor for both atherosclerosis and diastolic heart failure. Hypertens Res. 2004;27(9):625-631.

33. Meguro T, Nagatomo Y, Nagae A, Seki C, Kondou N, 
Shibata M, Oda Y. Elevated arterial stiffness evaluated by brachial-ankle pulse wave velocity is deleterious for the prognosis of patients with heart failure. Circ J. 2009;73(4):673-680.

34. Hitsumoto T. Arterial velocity pulse index as a novel marker of atherosclerosis using pulse wave analysis on high sensitivity troponin $\mathrm{T}$ in hypertensive patients. Cardiol Res. 2017;8(2):36-43.

35. Miyashita Y, Saiki A, Endo K, Ban N, Yamaguchi T, Kawana H, Nagayama D, et al. Effects of olmesartan, an angiotensin II receptor blocker, and amlodipine, a calcium channel blocker, on Cardio-Ankle Vascular Index (CAVI) in type 2 diabetic patients with hypertension. J Atheroscler Thromb. 2009;16(5):621-626.

36. Miyashita Y, Endo K, Saiki A, Ban N, Yamaguchi T, Kawana H, Nagayama D, et al. Effects of pitavastatin, a 3-hydroxy-3-methylglutaryl coenzyme a reductase inhibitor, on cardio-ankle vascular index in type 2 diabetic patients. J Atheroscler Thromb. 2009;16(5):539-545.

37. Tahara N, Yamagishi SI, Bekki M, Kodama N, Nakamura T, Sugiyama Y, Oshige T, et al. Anagliptin, a dipeptidyl peptidase-4 inhibitor ameliorates arterial stiffness in association with reduction of remnant-like particle cholesterol and alanine transaminase levels in type 2 diabetic patients. Curr Vasc Pharmacol. 2016;14(6):552-562.

38. Lodovici M, Bigagli E, Tarantini F, Di Serio C, Raimondi L. Losartan reduces oxidative damage to renal DNA and conserves plasma antioxidant capacity in diabetic rats. Exp Biol Med (Maywood). 2015;240(11):1500-1504.

39. Kakuda H, Kanasaki K, Koya D, Takekoshi N. The administration of pitavastatin augments creatinine clearance associated with reduction in oxidative stress parameters: acute and early effects. Clin Exp Nephrol. 2013;17(2):240-247.

40. Alam MA, Chowdhury MRH, Jain P, Sagor MAT, Reza HM. DPP-4 inhibitor sitagliptin prevents inflammation and oxidative stress of heart and kidney in two kidney and one clip (2K1C) rats. Diabetol Metab Syndr. 2015;7:107.

41. Iwasa T, Amiya E, Ando J, Watanabe M, Murasawa T, Komuro I. Different contributions of physical activity on arterial stiffness between diabetics and non-diabetics. PLoS One. 2016;11(8):e0160632. 